\title{
Relation-Theoretic Fixed Point Theorems for Generalized Weakly Contractive Mappings
}

\author{
Priyam Chakraborty ${ }^{1}$, Binayak S. Choudhury ${ }^{1}$ and Manuel De la Sen ${ }^{2, *}$ (i) \\ 1 Department of Mathematics, Indian Institute of Engineering Science and Technology, Shibpur, \\ Howrah-711103, India; priyam.math123@gmail.com (P.C.); binayak@math.iiests.ac.in (B.S.C.) \\ 2 Institute of Research and Development of Processes, University of the Basque Country, 48940 Leioa, \\ Bizkaia, Spain \\ * Correspondence: manuel.delasen@ehu.eus
}

Received: 15 November 2019; Accepted: 18 December 2019; Published: 21 December 2019

\begin{abstract}
In recent times there have been two prominent trends in metric fixed point theory. One is the use of weak contractive inequalities and the other is the use of binary relations. Combining the two trends, in this paper we establish a relation-theoretic fixed point result for a mapping which is defined on a metric space with an arbitrary binary relation and satisfies a weak contractive inequality for any pair of points whenever the pair of points is related by a given relation. The uniqueness is obtained by assuming some extra conditions. The metric space is assumed to be $\mathcal{R}$-complete. We use $\mathcal{R}$-continuity of functions. The property of local T-transitivity of the relation $\mathcal{R}$ is used in the main theorem. There is an illustrative example. An existing fixed point result is generalized through the present work. We use a method in the proof of our main theorem which is a blending of relation-theoretic and analytic approaches.
\end{abstract}

Keywords: relation; metric space; weak contraction; fixed point; $\mathcal{R}$-completeness

MSC: AMS Subject Classification 2000: 54H25

\section{Introduction and Preliminaries}

The present paper is a relation-theoretic fixed point result of a self-mapping on a metric space which is assumed to satisfy a weak contraction inequality. It is a combination of two recent trends of metric fixed point theory. One is the use of weak contractive inequalities to establish fixed point results while the other is to relax the set of pairs of points for which the contraction inequality is defined for the purpose of obtaining a fixed point result of the corresponding mapping. In the following we briefly dwell upon these two trends.

Weak contractions are intermediate concepts between a contraction mapping and a non-expansive mapping. They were first introduced by Alber et al. [1] in the context of Hilbert spaces and was subsequently defined in metric spaces by Rhoades [2]. In the same work, a weak contraction mapping principle was established. Afterwards, the idea was generalized and extended in several ways by which a large amount of literature on the fixed point theory of weakly contractive mappings was produced. Some instance of these works are [3-13].

On the other hand, it was realized that in several proofs of many important results of fixed point theory, it is not necessary to assume the contraction condition for arbitrary pairs of points chosen from the space itself. There can be suitable restrictions on the choice of such pairs. This perception had its expression in the fixed point theory on partially ordered metric space [14-19]. Also, there are several recent papers in fixed point theory on metric spaces with a graph, [20-22] for instance, where the contractive conditions are generally assumed for pairs of points which are adjacent vertices of the 
graph. Viewing graphs and partial orders as relations, attempts have been recently made to define weaker relations on metric spaces which will serve the same purpose. Focus on this line of research has been on binary relations on metric spaces which are weaker than graphs, etc.. Some works in this line are [23-27].

In the present context we prove a fixed point result for a self-mapping satisfying a weak contraction inequality for choices of points which are related by some relation having specific properties both in relation to the metric spaces and the function defined on it. We illustrate our result with an example. Our result is an extension of previous results existing in literature $[4,28]$.

In the extension, the following definitions will be utilized.

We denote $\mathbb{N}$ as the set of natural numbers. $\mathbb{R}$ as the set of real numbers.

Definition 1. [25] Let $X$ be any nonempty set and $\mathcal{R} \subseteq X \times X$ be some binary relation defined on $X$. Then two elements $u$ and $v$, of $X$, are $\mathcal{R}$ - comparative if either $(u, v) \in \mathcal{R}$ or $(v, u) \in \mathcal{R}$. This is denoted as $[u, v] \in \mathcal{R}$.

Definition 2. $[29,30]$ A binary relation $\mathcal{R}$ defined on any nonempty set $X$ is said to be

- $\quad$ reflexive if $(u, u) \in \mathcal{R}$ for all $u \in X$,

- $\quad$ symmetric if $(u, v) \in \mathcal{R}$ implies $(v, u) \in \mathcal{R}$,

- $\quad$ transitive if $(u, v) \in \mathcal{R},(v, w) \in \mathcal{R}$ implies $(u, w) \in \mathcal{R}$,

- dichotomous if $[u, v] \in \mathcal{R}$ for all $u, v \in X$,

- $\quad$ trichotomous if $[u, v] \in \mathcal{R}$ or $u=v$ for all $u, v \in X$,

- equivalence if $\mathcal{R}$ is reflexive, symmetric and transitive.

Definition 3. [29] Let $X$ be any nonempty set with a binary relation $\mathcal{R}$ on $X$.

(1) The inverse or dual relation of $\mathcal{R}$, denoted by $\mathcal{R}^{-1}$, is defined by

$$
\mathcal{R}^{-1}=\{(u, v) \in X \times X:(v, u) \in \mathcal{R}\} .
$$

(2) The reflexive closure of $\mathcal{R}$, denoted by $\mathcal{R}^{\#}$, is defined as $\mathcal{R}^{\#}:=\mathcal{R} \cup \triangle_{u}$, where $\triangle_{u}=\{(u, u): u \in X\}$.

(3) The symmetric closure of $\mathcal{R}$, denoted by $\mathcal{R}^{s}$, is defined to be $\mathcal{R}^{s}:=\mathcal{R} \cup \mathcal{R}^{-1}$.

Remark 1. $\mathcal{R}^{\#}$ is the smallest relation on $X$, containing $\mathcal{R}$, which is reflexive. $\mathcal{R}^{s}$ is the smallest symmetric relation on $X$ containing $\mathcal{R}$.

Definition 4. $\mathcal{R}$-preserving sequence: [31] Let $X$ be any nonempty set with a binary relation $\mathcal{R}$ on it. We call the sequence $\left\{u_{n}\right\} \subset X$ to be $\mathcal{R}$-preserving if $\left(u_{n}, u_{n+1}\right) \in \mathcal{R}$ for all $n \in \mathbb{N}_{0}$.

Definition 5. $d$-self closed relation: [25] Let $(X, d)$ be a metric space with a binary relation $\mathcal{R}$ on it. $\mathcal{R}$ is $d$-self closed if for any $\mathcal{R}$-preserving sequence $\left\{u_{n}\right\}$ with $u_{n} \longrightarrow u$, there exists a subsequence $\left\{u_{n_{k}}\right\}$ of $\left\{u_{n}\right\}$ with $\left[u_{n_{k}}, u\right] \in \mathcal{R}$ for every $k \in \mathbb{N}_{0}$.

Definition 6. T-closed relation: [25] Let $X$ be any nonempty set with the self-map $T: X \rightarrow X . A$ binary relation $\mathcal{R}$ on $X$ is called $T$-closed if for any $u, v \in X,(u, v) \in \mathcal{R} \Longrightarrow(T u, T v) \in \mathcal{R}$.

Definition 7. $\mathcal{R}$-directed set: [32] Let $X$ be a nonempty set with a binary relation $\mathcal{R}$ on it. The set $E \subseteq X$ is called $\mathcal{R}$-directed if for each $u, v \in E$, there exists $w \in X$ such that $(u, w) \in \mathcal{R}$ and $(v, w) \in \mathcal{R}$. 
Definition 8. Path of length $k$ : [33] Let $X$ be a nonempty set with a binary relation $\mathcal{R}$ on it. For $u, v \in X$, a path of length $k$ ( where $k$ is a natural number) in $\mathcal{R}$ from $u$ to $v$ is a finite sequence $\left\{z_{0}, z_{1}, z_{2}, \ldots, z_{k}\right\} \subset X$ with the following conditions:

$$
\text { (i) } z_{0}=u \text { and } z_{k}=v \text {, }
$$

(ii) $\left(z_{i}, z_{i+1}\right) \in \mathcal{R}$ for each $i$, where $(0 \leq i \leq k-1)$.

A path of length $k$ contains $k+1$ elements of $X$ which may be repeated.

Definition 9. Transitive relation: [26] A nonempty subset $\mathcal{R}$ of $X \times X$ is transitive if $(u, w) \in \mathcal{R}$ for all $u, v, w \in X$ such that $(u, v),(v, w) \in \mathcal{R}$.

Definition 10. T-Transitive relation: [26] Given a map $T: X \rightarrow X$, we say a relation $\mathcal{R}$ on $X$ to be $T$ -transitive if $(T u, T w) \in \mathcal{R}$ for all $u, v, w \in X$ such that $(T u, T v),(T v, T w) \in \mathcal{R}$.

Definition 11. Locally Transitive relation: [26] A binary relation $\mathcal{R}$ on a nonempty set $X$ is said to be locally transitive if for every (effectively) $\mathcal{R}$-preserving sequence $\left\{u_{n}\right\} \subseteq X$ (with range $E:=\left\{u_{n}: n \in \mathbb{N}\right\}$ ), the relation $\left.R\right|_{E}$ is transitive.

Definition 12. Locally T-Transitive relation: [26] Let $X$ be a nonempty set with a self-mapping $T$ on it. A binary relation $\mathcal{R}$ on $X$ is said to be locally $T$-transitive if for every (effectively) $\mathcal{R}$-preserving sequence $\left\{u_{n}\right\} \subseteq T(X)$ (with range $E:=\left\{u_{n}: n \in \mathbb{N}\right\}$ ), the binary relation $\left.R\right|_{E}$ is transitive.

Definition 13. T-symmetric relation: Given a self mapping $T: X \rightarrow X$, we say that a binary relation $\mathcal{R}$ on $X$ is $T$-symmetrie if $(T u, T v) \in \mathcal{R}$ for all $u, v \in X$ such that $(T v, T u) \in \mathcal{R}$.

Definition 14. $\mathcal{R}$-complete: [24] Let $(X, d)$ be a metric space with a binary relation $\mathcal{R}$ on it. We say that $(X, d)$ is $\mathcal{R}$-complete if every $\mathcal{R}$-preserving Cauchy sequence in $X$ is convergent.

Definition 15. $\mathcal{R}$-closed: [24] Let $(X, d)$ be a metric space and $\mathcal{R}$ a binary relation on it. The set $E \subseteq X$ is said to be $\mathcal{R}$-closed if every $\mathcal{R}$-preserving convergent sequence in $E$ is convergent to a point of $E$.

Definition 16. $\mathcal{R}$-continuous function: [24] Let $(X, d)$ be a metric space with a binary relation $\mathcal{R}$ on it and $u \in X$. A mapping $f: X \rightarrow X$ is called $\mathcal{R}$-continuous at the point $u$ if for every $\mathcal{R}$-preserving sequence $\left\{u_{n}\right\}$, $u_{n} \rightarrow u \Rightarrow f u_{n} \rightarrow f u$.

$f$ is called $\mathcal{R}$-continuous if the function $f$ is $\mathcal{R}$-continuous at every point of $X$.

Definition 17. $\mathcal{R}$-connected set: [26] Let $X$ be a non empty set with a binary relation $\mathcal{R}$ on it. A subset $E$ of $X$ is $\mathcal{R}$-connected if there is a path from $u$ to $v$ in $\mathcal{R}$, for all $u, v \in E$.

In this paper if $T: X \rightarrow X$ is a self-mapping and $\mathcal{R}$ is a binary relation on it, we use the notations:

$$
\begin{aligned}
X(T ; \mathcal{R}) & :=\{u \in X:(u, T u) \in \mathcal{R}\} \\
\mathbb{N}_{0} & :=\mathbb{N} \cup\{0\} .
\end{aligned}
$$

\section{Main Results}

Theorem 1. Let $(X, d)$ be a metric space with some binary relation $\mathcal{R}$ on it and $T: X \rightarrow X$ be a self map, with the following conditions satisfied.

(i) $(X, d)$ is $\mathcal{R}$-complete,

(ii) $X(T, \mathcal{R}) \neq \varnothing$ 
(iii) $\mathcal{R}$ is $T$-closed

(iv) $\mathcal{R}$ is locally $T$-transitive

(v) either $T$ is $\mathcal{R}$-continuous or $\mathcal{R}$ is d-selfclosed,

(vi) $T$ is a generalized weakly contractive map on $\mathcal{R}$, i.e.,

there exist two continuous functions $\phi, \psi:[0, \infty) \rightarrow[0, \infty)$, such that $\phi(t)=\psi(t)=0$ if and only if $t=0$, and if $x, y \in X$ with $[x, y] \in \mathcal{R}$ then

$$
\psi(d(T x, T y)) \leq \psi\left(m_{1}(x, y)\right)-\phi\left(m_{2}(x, y)\right)
$$

where $m_{1}(x, y):=\max \left\{d(x, y), d(x, T x), d(y, T y), \frac{1}{2}[d(x, T y)+d(y, T x)]\right\}$

and $m_{2}(x, y):=\max \{d(x, y), d(y, T y)\}$,

then $T$ has a fixed point.

Proof. As $X(T, \mathcal{R}) \neq \varnothing, \exists x_{0} \in X$ such that $\left(x_{0}, T x_{0}\right) \in \mathcal{R}$.

Let us define a sequence $\left\{x_{n}\right\}$ by $x_{n}:=T^{n} x_{0}$.

Since $\mathcal{R}$ is $T$-closed and $\left(x_{0}, T x_{0}\right) \in \mathcal{R}$, we have $\forall i \in \mathbb{N},\left(T^{i} x_{0}, T^{i+1} x_{0}\right) \in \mathcal{R}$, that is, $\left(x_{i}, x_{i+1}\right) \in \mathcal{R}, \forall i \in \mathbb{N}$.

Therefore $\left\{x_{n}\right\}$ is an $\mathcal{R}$-preserving sequence.

Let $\rho_{n}:=d\left(x_{n}, x_{n+1}\right)$.

From the contractive condition in Equation (1) we have, for all $n \in \mathbb{N}_{0}$

$$
\begin{aligned}
\psi\left(d\left(T x_{n}, T x_{n+1}\right)\right) & \leq \psi\left(m_{1}\left(x_{n}, x_{n+1}\right)\right)-\phi\left(m_{2}\left(x_{n}, x_{n+1}\right)\right) \\
\text { or } \quad \psi\left(\rho_{n+1}\right) & \leq \psi\left(m_{1}\left(x_{n}, x_{n+1}\right)\right)-\phi\left(m_{2}\left(x_{n}, x_{n+1}\right)\right),
\end{aligned}
$$

where $m_{1}\left(x_{n}, x_{n+1}\right)=\max \left\{d\left(x_{n}, x_{n+1}\right), d\left(x_{n+1}, x_{n+1}\right), d\left(x_{n+1}, x_{n+2}\right)\right.$,

$$
\left.\frac{1}{2}\left(d\left(x_{n}, x_{n+2}\right)+d\left(x_{n+1}, x_{n+1}\right)\right)\right\} \text {. }
$$

and $m_{2}\left(x_{n}, x_{n+1}\right)=\max \left\{d\left(x_{n}, x_{n+1}\right), d\left(x_{n+1}, x_{n+2}\right)\right\}$.

Again we have, for all $n \in \mathbb{N}_{0}, \quad d\left(x_{n}, x_{n+2}\right) \leq d\left(x_{n}, x_{n+1}\right)+d\left(x_{n+1}, x_{n+2}\right)$ $\leq 2 \max \left\{d\left(x_{n}, x_{n+1}\right), d\left(x_{n+1}, x_{n+2}\right)\right\}$,

that is, $\frac{1}{2}\left(d\left(x_{n}, x_{n+2}\right)+d\left(x_{n+1}, x_{n+1}\right)\right) \leq \max \left\{d\left(x_{n}, x_{n+1}\right), d\left(x_{n+1}, x_{n+2}\right)\right\}$.

Thus $m_{1}\left(x_{n}, x_{n+1}\right)=\max \left\{\rho_{n}, \rho_{n+1}\right\}, \forall n \in \mathbb{N}_{0}$.

Then Equation (2) reduces to

$$
\psi\left(\rho_{n+1}\right) \leq \psi\left(\max \left\{\rho_{n}, \rho_{n+1}\right\}\right)-\phi\left(\max \left\{\rho_{n}, \rho_{n+1}\right\}\right) .
$$

\section{Case-I :}


If $\rho_{n_{0}}<\rho_{n_{0}+1}$, for some $n_{0} \in \mathbb{N}$, then from Equation (3) we have

$$
\begin{aligned}
& \psi\left(\rho_{n_{0}+1}\right) \leq \psi\left(\rho_{n_{0}+1}\right)-\phi\left(\rho_{n_{0}+1}\right) \\
& \Rightarrow \phi\left(\rho_{n_{0}+1}\right)=0 \\
& \Rightarrow \rho_{n_{0}+1}=0 \quad[\text { as } \phi(t)=0 \Leftrightarrow t=0] \\
& \Rightarrow d\left(x_{n_{0}+1}, x_{n_{0}+2}\right)=0 \\
& \Rightarrow x_{n_{0}+1}=x_{n_{0}+2}=T x_{n_{0}+1} .
\end{aligned}
$$

In this case $x_{n_{0}+1}$ is a fixed point of $T$ and we arrive at the conclusion of the theorem.

\section{Case-II :}

If $\rho_{n} \geq \rho_{n+1} \forall n \in \mathbb{N}$, then $\left\{\rho_{n}\right\}$ is a sequence of non-negative real numbers which is monotone decreasing.

The sequence $\left\{\rho_{n}\right\}$ is bounded below by 0 and, as it is monotone decreasing, it must be convergent.

Let $\lim _{n \rightarrow \infty} \rho_{n}=\rho$ (say).

In this case, from (3) we have $\psi\left(\rho_{n+1}\right) \leq \psi\left(\rho_{n}\right)-\phi\left(\rho_{n}\right)$.

Taking limit $n \rightarrow \infty$ and using continuity of the functions $\phi$ and $\psi$, we have $\psi(\rho) \leq \psi(\rho)-\phi(\rho)$, which implies that $\phi(\rho)=0$.

Then, by a property of $\phi$,we have $\rho=0$, that is, $\lim _{n \rightarrow \infty} d\left(x_{n}, x_{n+1}\right)=0$.

We will now establish that $\left\{x_{n}\right\}$ is a Cauchy sequence.

Otherwise, there exists some $\epsilon>0$ for which we can find two sequences of positive integers $\left\{m_{k}\right\}$ and $\left\{n_{k}\right\}$ such that for every positive integer $k$,

$$
\begin{aligned}
& n_{k}>m_{k}>k, \\
& d\left(x_{m_{k}}, x_{n_{k}}\right) \geq \epsilon \text { and } d\left(x_{m_{k}}, x_{n_{k}-1}\right)<\epsilon .
\end{aligned}
$$

Now, using (4) we get, $\epsilon \leq d\left(x_{m_{k}}, x_{n_{k}}\right)<\epsilon+d\left(x_{n_{k}-1}, x_{n_{k}}\right)$.

Taking the limit as $k \longrightarrow \infty$ in the above and using the result proved above that $\lim _{n \rightarrow \infty} d\left(x_{n}, x_{n+1}\right)=0$, $\forall n \in \mathbb{N}$, we have

$$
\lim _{k \rightarrow \infty} d\left(x_{m_{k}}, x_{n_{k}}\right)=\epsilon
$$

Also,

$$
d\left(x_{m_{k}}, x_{n_{k}+1}\right) \leq d\left(x_{m_{k}}, x_{n_{k}}\right)+d\left(x_{n_{k}}, x_{n_{k}+1}\right) .
$$

Taking the limit $k \longrightarrow \infty$ in the above,

$$
\lim _{k \rightarrow \infty} d\left(x_{m_{k}}, x_{n_{k}+1}\right) \leq \epsilon+0
$$

Also

$$
d\left(x_{m_{k}}, x_{n_{k}}\right) \leq d\left(x_{m_{k}}, x_{n_{k}+1}\right)+d\left(x_{n_{k}+1}, x_{n_{k}}\right) .
$$

Taking the limit $k \longrightarrow \infty$ in the above inequalities, we obtain $\epsilon \leq \lim _{k \rightarrow \infty} d\left(x_{m_{k}}, x_{n_{k}+1}\right)+0$.

$$
\text { Thus } \quad \lim _{k \rightarrow \infty} d\left(x_{m_{k}}, x_{n_{k}+1}\right)=\epsilon \text {. }
$$

Also

$$
d\left(x_{m_{k}+1}, x_{n_{k}}\right) \leq d\left(x_{m_{k}+1}, x_{m_{k}}\right)+d\left(x_{m_{k}}, x_{n_{k}}\right)
$$


Taking the limit as $k \longrightarrow \infty$ in the above inequalities, we obtain

$$
\lim _{k \rightarrow \infty} d\left(x_{m_{k}+1}, x_{n_{k}}\right) \leq \epsilon
$$

Also

$$
d\left(x_{m_{k}}, x_{n_{k}}\right) \leq d\left(x_{m_{k}}, x_{m_{k}+1}\right)+d\left(x_{m_{k}+1}, x_{n_{k}}\right)
$$

Taking the limit as $k \longrightarrow \infty$ in the above inequalities, we have

$$
\epsilon \leq \lim _{k \rightarrow \infty} d\left(x_{m_{k}+1}, x_{n_{k}}\right)
$$

Thus $\quad \lim _{k \rightarrow \infty} d\left(x_{m_{k}+1}, x_{n_{k}}\right)=\epsilon$.

Again,

$$
d\left(x_{m_{k}+1}, x_{n_{k}+1}\right) \leq d\left(x_{m_{k}+1}, x_{m_{k}}\right)+d\left(x_{m_{k}}, x_{n_{k}+1}\right) .
$$

Taking the limit as $k \longrightarrow \infty$ in the above inequalities and using (6), we have

$$
\lim _{k \rightarrow \infty} d\left(x_{m_{k}+1}, x_{n_{k}+1}\right) \leq \epsilon
$$

Also

$$
d\left(x_{m_{k}+1}, x_{n_{k}}\right) \leq d\left(x_{m_{k}+1}, x_{n_{k}+1}\right)+d\left(x_{n_{k}+1}, x_{n_{k}}\right) .
$$

Taking the limit as $k \longrightarrow \infty$ in the above inequalities and using (7), we have

$$
\epsilon \leq \lim _{k \rightarrow \infty} d\left(x_{m_{k}+1}, x_{n_{k}+1}\right)
$$

Thus $\quad \lim _{k \rightarrow \infty} d\left(x_{m_{k}+1}, x_{n_{k}+1}\right)=\epsilon$.

Now, as $\left\{x_{n}\right\}$ is $\mathcal{R}$ preserving sequence and by the local $T$-transitivity of $\mathcal{R}$, we have $\left(x_{i}, x_{j}\right) \in$ $\mathcal{R} \forall i<j$. Thus we can say that $\left[x_{m_{k}}, x_{n_{k}}\right] \in \mathcal{R}$ for any $k \in \mathbb{N}$.

Therefore, from (1) $\psi\left(d\left(x_{m_{k}+1}, x_{n_{k}+1}\right)\right) \leq \psi\left(m_{1}\left(x_{m_{k}}, x_{n_{k}}\right)\right)-\phi\left(m_{2}\left(x_{m_{k}}, x_{n_{k}}\right)\right)$.

Taking limit as $k \rightarrow \infty$ and using continuity of $\phi$ and $\psi$ we have $\psi(\epsilon) \leq \psi(\epsilon)-\phi(\epsilon)$, that is $\phi(\epsilon)=0$, which, by a property of $\phi$, implies that $\epsilon=0$, this contradicts the fact that $\epsilon>0$.

Hence $\left\{x_{n}\right\}$ is $\mathcal{R}$-preserving Cauchy sequence in $X$.

Thus $\left\{x_{n}\right\} \longrightarrow x$ for some $x \in X$, as $X$ is $\mathcal{R}$-complete.

Now we consider two subcases,

Subcase-II(A): If $T$ is $\mathcal{R}$-continuous, then using the fact $\left\{x_{n}\right\} \longrightarrow x$ in both sides of $x_{n+1}=T x_{n}$, we have $x=T x$.

Thus $x$ is a fixed point.

Subcase-II(B): If $T$ is not $\mathcal{R}$-continuous, then by a condition of the theorem, $\mathcal{R}$ is $d$-self closed.

Then for the $\mathcal{R}$-preserving sequence $\left\{x_{n}\right\}$, where $\lim _{n \rightarrow \infty} x_{n}=x, \exists$ a subsequence $\left\{x_{n_{k}}\right\}$ of $\left\{x_{n}\right\}$ such that $\left[x_{n_{k}}, x\right] \in \mathcal{R}$ for all $k \in \mathbb{N}$.

Then we have, $\psi\left(d\left(T x_{n_{k}}, T x\right)\right) \leq \psi\left(m_{1}\left(x_{n_{k}}, x\right)\right)-\phi\left(m_{2}\left(x_{n_{k}}, x\right)\right)$

$$
\text { or, } \psi\left(d\left(x_{n_{k}+1}, T x\right)\right) \leq \psi\left(m_{1}\left(x_{n_{k}}, x\right)\right)-\phi\left(m_{2}\left(x_{n_{k}}, x\right)\right) \text {, }
$$




$$
\begin{aligned}
\text { where } m_{1}\left(x_{n_{k}}, x\right) & =\max \left\{d\left(x_{n_{k}}, x\right), d\left(x_{n_{k}}, T x_{n_{k}}\right), d(x, T x), \frac{1}{2}\left(d\left(x_{n_{k}}, T x\right)+d\left(T x_{n_{k}}, x\right)\right)\right\} \\
& =\max \left\{d\left(x_{n_{k}}, x\right), d\left(x_{n_{k}}, x_{n_{k}+1}\right), d(x, T x), \frac{1}{2}\left(d\left(x_{n_{k}}, T x\right)+d\left(x_{n_{k}+1}, x\right)\right)\right\} \\
m_{2}\left(x_{n_{k}}, x\right) & =\max \left\{d\left(x_{n_{k}}, x\right), d(x, T x)\right\} .
\end{aligned}
$$

Now

$$
\begin{aligned}
\lim _{k \rightarrow \infty} \psi\left(d\left(x_{n_{k}+1}, T x\right)\right) & =\psi\left(\lim _{k \rightarrow \infty} d\left(x_{n_{k}+1}, T x\right)\right) \quad \text { [as } \psi \text { is continuous] } \\
& =\psi(d(x, T x))
\end{aligned}
$$

$$
\begin{aligned}
\lim _{k \rightarrow \infty} \psi\left(m_{1}\left(x_{n_{k}}, x\right)\right) & =\psi\left(\lim _{k \rightarrow \infty} m_{1}\left(x_{n_{k}}, x\right)\right) \quad \text { [as } \psi \text { is continuous] } \\
& =\psi\left(\lim _{k \rightarrow \infty} \max \left\{d\left(x_{n_{k}}, x\right), d\left(x_{n_{k}}, x_{n_{k}+1}\right), d(x, T x), \frac{1}{2}\left(d\left(x_{n_{k}}, T x\right)+d\left(x_{n_{k}+1}, x\right)\right)\right\}\right) \\
& =\psi\left(\operatorname { m a x } \left\{\lim _{k \rightarrow \infty} d\left(x_{n_{k}}, x\right), \lim _{k \rightarrow \infty} d\left(x_{n_{k}}, x_{n_{k}+1}\right), \lim _{k \rightarrow \infty} d(x, T x),\right.\right. \\
& \left.\left.\frac{1}{2} \lim _{k \rightarrow \infty}\left(d\left(x_{n_{k}}, T x\right)+d\left(x_{n_{k}+1}, x\right)\right)\right\}\right) \\
& =\psi\left(\max \left\{d(x, x), d(x, x), d(x, T x), \frac{1}{2}(d(x, T x)+d(x, x))\right\}\right) \\
& =\psi(d(x, T x))
\end{aligned}
$$

and

$$
\begin{aligned}
\lim _{k \rightarrow \infty} \phi\left(m_{1}\left(x_{n_{k}}, x\right)\right) & =\phi\left(\lim _{k \rightarrow \infty} m_{1}\left(x_{n_{k}}, x\right)\right) \quad \text { [as } \phi \text { is continuous] } \\
& =\phi\left(\lim _{k \rightarrow \infty} \max \left\{d\left(x_{n_{k}}, x\right), d(x, T x)\right\}\right) \\
& =\phi\left(\max \left\{\lim _{k \rightarrow \infty} d\left(x_{n_{k}}, x\right), \lim _{k \rightarrow \infty} d(x, T x)\right\}\right) \\
& =\phi(\max \{d(x, x), d(x, T x)\}) \\
& =\phi(d(x, T x)) .
\end{aligned}
$$

Taking limit as $k \rightarrow \infty$ in both sides of (9) we have,

$$
\begin{array}{ll}
\lim _{k \rightarrow \infty} \psi\left(d\left(x_{n_{k}+1}, T x\right)\right) \leq \lim _{k \rightarrow \infty} \psi\left(m_{1}\left(x_{n_{k}}, x\right)\right)-\lim _{k \rightarrow \infty} \phi\left(m_{2}\left(x_{n_{k}}, x\right)\right) \\
\text { or, } \quad \psi(d(x, T x)) \leq \psi(d(x, T x))-\phi(d(x, T x)) \\
\text { or, } \quad \phi(d(x, T x))=0 \\
\text { or, } \quad d(x, T x)=0 \\
\text { or, } \quad x=T x .
\end{array}
$$

Thus also in this subcase $x$ is a fixed point.

\section{Note:}

(i) In Theorem 1, the generalized weak contraction appearing through inequality (1) has been considered in [4] for the case of complete metric spaces without any ordering. Here we prove the existence of fixed points of these contractions with some variations in a metric space, having a binary relation $\mathcal{R}$. Later in the last section we describe an example which shows that the result of [4], is effectively generalized here.

(ii) In Subcase II-B of the proof we use Definition 11 of $d$-self closed relation given by Alam et al. 
in [25]. This is actually weaker than that given by Turinici [31] for the partial order case. If we consider the definition of Turinici [31], then there is no need to consider subsequences.

Remark 2. Fixed point results for weakly contractive mappings have more scopes of applications than the corresponding results for contractive mappings in a natural way. In the above theorem, results are obtained under a further relaxation by correlating the weak contraction condition with a binary relation. This widens the scope of applications to a large extent.

For uniqueness of the fixed point we need some further conditions.

Theorem 2. In addition to the conditions stated in Theorem 1, if any of the following conditions hold

(vii.a) $T(X)$ is $\mathcal{R}$-connected and if $x_{0}$ is a fixed point of $T$, then $x_{0} \in X(T, \mathcal{R})$.

(vii.b) $T(X)$ is $\mathcal{R}$-connected and $\mathcal{R}$ is $T$-transitive.

(vii.c) $T(X)$ is $\mathcal{R}$-connected and $\mathcal{R}$ is $T$-symmetric.

Then the fixed point is unique.

Proof. Let (vii.a) hold.

Let $x, y$ be two fixed points of $T$.

Since $T$ is $\mathcal{R}$-connected, there is a finite path $\left\{z_{0}, z_{1}, \ldots, z_{k_{1}}\right\}$ such that $x=z_{0}, y=z_{k_{1}}, z_{i} \in T(X)$ and $\left(z_{i}, z_{i+1}\right) \in \mathcal{R}$ for $0 \leq i \leq k_{1}-1$.

We construct a sequence $x_{n}$ as follows

$$
x_{1}=x=z_{0}, x_{2}=z_{1}, \ldots, x_{k_{1}+1}=z_{k_{1}}=y \text { and } x_{n}=y, \text { for } n \geq k_{1}+2 .
$$

Since $y$ is a fixed point of $T, y \in X(T, \mathcal{R})$ or $(y, T y)=(y, y) \in \mathcal{R}$.

Hence $\left\{x_{n}\right\}$ is a $\mathcal{R}$-preserving sequence.

By assumption (iv) of Theorem $\mathbf{1},(x, y) \in \mathcal{R}$, as $\mathcal{R}$ is locally $T$-transitive.

Now by assumption (vi) of Theorem 1, it follows that,

$$
\begin{aligned}
& \psi(d(T x, T y)) \leq \psi\left(m_{1}(x, y)\right)-\phi\left(m_{2}(x, y)\right) \\
& \text { where } m_{1}(x, y)=\max \left\{d(x, y), d(x, T x), d(y, T y), \frac{1}{2}[d(x, T y)+d(y, T x)]\right\} \\
& =\max \left\{d(x, y), d(x, x), d(y, y), \frac{1}{2}[d(x, y)+d(y, x)]\right\} \\
& =d(x, y) \\
& \text { and } m_{2}(x, y)=\max \{d(x, y), d(y, T y)\} \text {, } \\
& =\max \{d(x, y), d(y, y)\}, \\
& =d(x, y) \text {. }
\end{aligned}
$$

From (12) we have

$$
\begin{gathered}
\psi(d(x, y)) \leq \psi(d(x, y))-\phi(d(x, y)) \\
\text { or, } \phi(d(x, y))=0 \\
\text { or, } d(x, y)=0 \\
\text { or, } x=y .
\end{gathered}
$$


This proves the uniqueness of the fixed point.

Now let (vii.b) hold.

Let us assume there be two fixed points $x, y$ of $T$.

Since $T$ is $\mathcal{R}$-connected, there is a finite path $\left\{z_{0}, z_{1}, \ldots, z_{k_{2}}\right\}$ such that $x=z_{0}, y=z_{k_{2}}, z_{i} \in T(X)$ and $\left(z_{i}, z_{i+1}\right) \in \mathcal{R}$ for $0 \leq i \leq k_{2}-1$.

As $\mathcal{R}$ is $T$-transitive, $(x, y) \in \mathcal{R}$.

Now by assumption (vi) of Theorem 1, we have,

$$
\begin{aligned}
& \begin{aligned}
\psi(d(T x, T y)) & \leq \psi\left(m_{1}(x, y)\right)-\phi\left(m_{2}(x, y)\right) \\
\text { where } m_{1}(x, y) & =\max \left\{d(x, y), d(x, T x), d(y, T y), \frac{1}{2}[d(x, T y)+d(y, T x)]\right\} \\
= & \max \left\{d(x, y), d(x, x), d(y, y), \frac{1}{2}[d(x, y)+d(y, x)]\right\} \\
= & d(x, y)
\end{aligned} \\
& \text { and } \begin{aligned}
m_{2}(x, y) & =\max \{d(x, y), d(y, T y)\}, \\
= & \max \{d(x, y), d(y, y)\} \\
= & d(x, y) .
\end{aligned}
\end{aligned}
$$

From (15) we have

$$
\begin{gathered}
\psi(d(x, y)) \leq \psi(d(x, y))-\phi(d(x, y)) \\
\text { or, } \phi(d(x, y))=0 \\
\text { or, } d(x, y)=0 \\
\text { or, } x=y .
\end{gathered}
$$

Thus the fixed point is unique in this case also.

Now let (vii.c) hold.

Let $x, y$ be two fixed points of $T$.

Since $T$ is $\mathcal{R}$-connected, there is a finite path $\left\{z_{0}, z_{1}, \ldots, z_{k_{3}}\right\}$ such that $x=z_{0}, y=z_{k_{3}}$, $z_{i} \in T(X)$ and $\left(z_{i}, z_{i+1}\right) \in \mathcal{R}$ for $0 \leq i \leq k_{3}-1$.

We construct a sequence $x_{n}$ as follows

$x_{1}=x=z_{0}, x_{2}=z_{1}, \ldots, x_{k_{3}}=z_{k_{3}-1}, x_{k_{3}+1}=z_{k_{3}}=y$ and $x_{k_{3}+(2 i-1)}=z_{k_{3}}, x_{k_{3}+2 i}=$ $z_{k_{3}-1}$, for all $i \in \mathbb{N}$.

As $\mathcal{R}$ is $T$-symmetric, i.e., $\mathcal{R}$ is symmetric on $T(X)$ we have $\left(z_{k_{3}-1}, z_{k_{3}}\right) \in \mathcal{R} \Rightarrow\left(z_{k_{3}}, z_{k_{3}-1}\right) \in \mathcal{R}$. Hence $\left\{x_{n}\right\}$ is a $\mathcal{R}$-preserving sequence and as $\mathcal{R}$ is locally $T$-transitive, we have $(x, y) \in \mathcal{R}$.

Now by assumption (vi) of Theorem 1, we have,

$$
\begin{aligned}
& \begin{aligned}
\psi(d(T x, T y)) & \leq \psi\left(m_{1}(x, y)\right)-\phi\left(m_{2}(x, y)\right) \\
\text { where } m_{1}(x, y) & =\max \left\{d(x, y), d(x, T x), d(y, T y), \frac{1}{2}[d(x, T y)+d(y, T x)]\right\} \\
= & \max \left\{d(x, y), d(x, x), d(y, y), \frac{1}{2}[d(x, y)+d(y, x)]\right\} \\
= & d(x, y)
\end{aligned} \\
& \text { and } \begin{aligned}
m_{2}(x, y) & =\max \{d(x, y), d(y, T y)\} \\
= & \max \{d(x, y), d(y, y)\} \\
= & d(x, y) .
\end{aligned}
\end{aligned}
$$


From (18) we have

$$
\begin{gathered}
\psi(d(x, y)) \leq \psi(d(x, y))-\phi(d(x, y)) \\
\text { or, } \phi(d(x, y))=0 \\
\text { or, } d(x, y)=0 \\
\text { or, } x=y .
\end{gathered}
$$

Thus in this case also, we find that the fixed point would be unique.

Hence our result is proved.

Remark 3. In Theorem 2 some additional conditions ensure the uniqueness of the fixed point. This creates the additional feature that whatever be the admissible initial choice of $x_{0}$, the algorithm developed here converges to unique fixed point. This creates an operational advantage in possible applications of the theorem.

Further we note that the above theorems combine two trends of research in metric fixed point theory, one in which contractive conditions are weakened in a particular way by using weak inequalities and the other in which restricting the relevant pairs of points with respect to which contractive conditions are to be satisfied through the introduction of appropriate relations. In both cases, the aim is to retain the fixed point property of the relevant function under relaxed conditions. Some instances of works in the former line are [3-5,9-11] while references [23-26] are some works obtained in the latter line of research. Particularly, the present work directly extends the result of [4].

\section{Illustration}

Example 1. Let $X=\mathbb{R}$. Let $N_{0}=\{0,1,2,3, \ldots\}$.

Let $d: X \times X \longrightarrow \mathbb{R}$ be given as

$$
d(u, v)=\left\{\begin{array}{l}
|u|+|v|, \text { if } u \neq v, \\
0, \text { otherwise }
\end{array}\right.
$$

Then $(X, d)$ is a metric space.

Let $T: X \longrightarrow X$ be a self-map, defined as follows:

$$
T u=\left\{\begin{array}{l}
u-1, \text { if } u \neq 0, \\
0, \quad \text { if } u=0 .
\end{array}\right.
$$

Consider the relation $\mathcal{R}=N_{0} \times N_{0}$ on $X$.

Clearly $(X, d)$ is $\mathcal{R}$-complete.

We also have $(2, T(2))=(2,2-1)=(2,1) \in \mathcal{R}$ i.e., $2 \in X(T, \mathcal{R})$.

So, $X(T, \mathcal{R}) \neq \varnothing$.

$$
\text { Let }(u, v) \in \mathcal{R} \text {. }
$$

If $u, v \in N_{0}-\{0\}$ then $(u-1, v-1) \in \mathcal{R}$.

If $u=0, v \neq 0$, and $u, v \in N_{0}$, then $(T u, T v)=(0, v-1) \in \mathcal{R}$.

If $u \neq 0, v=0$, and $u, v \in N_{0}$, then $(T u, T v)=(u-1,0) \in \mathcal{R}$.

If $u=0, v=0$, and $u, v \in N_{0}$, then $(T u, T v)=(0,0) \in \mathcal{R}$.

Thus for all $(u, v) \in \mathcal{R},(T u, T v) \in \mathcal{R}$.

Hence $\mathcal{R}$ is $T$-closed.

Also, the given relation $\mathcal{R}$ is Transitive and hence locally T-transitive. 
Next we note that if $\left\{u_{n}\right\}$ is a $\mathcal{R}$-preserving sequence converging to $u$, then it is eventually constant sequence. So, for a $\mathcal{R}$-preserving sequence, $\left\{u_{n}\right\}$, if $u_{n} \longrightarrow u$ as $n \longrightarrow \infty$, then $\exists k \in \mathbb{N}$ such that $u_{n}=u, \forall n \geq k$.

Thus, as $n \longrightarrow \infty, T u_{n}=u_{n}-1 \longrightarrow u-1$, if $u \neq 0$, and $T u_{n} \longrightarrow 0$, if $u=0$.

Thus for any $\mathcal{R}$-preserving sequence, $\left\{u_{n}\right\}, u_{n} \longrightarrow u \Rightarrow T u_{n} \longrightarrow T u$.

Hence $T$ is $\mathcal{R}$-continuous.

Let $\psi:[0, \infty) \longrightarrow[0, \infty)$ be as follows:

$\psi(t)=t^{2}, t \geq 0$.

Let $\phi:[0, \infty) \longrightarrow[0, \infty)$ be as follows:

for $s \in[0, \infty)$,

$$
\phi(t)= \begin{cases}\frac{t^{2}}{2}, & \text { if } t \leq 1, \\ \frac{1}{2}, & \text { if } t>1 .\end{cases}
$$

Then $\psi$ and $\phi$ are continuous and $\psi(t)=\phi(t)=0$ if and only if $t=0$.

To verify condition (vi) of Theorem 1, let us consider the following cases for $u, v \in X$ with $(u, v) \in \mathcal{R}$

Case-I: $u \neq v$.

(i) If $v \neq 0$ and $u>v$, then

$\psi(d(T u, T v))=\psi(d(u-1, v-1))=\psi(u+v-2)=(u+v-2)^{2}$.

$\psi\left(m_{1}(u, v)\right)=\psi\left(\max \left\{d(u, v), d(u, T u), d(v, T v), \frac{1}{2}[d(u, T v)+d(v, T u)]\right\}\right)$

$=\psi\left(\max \left\{u+v, 2 u-1,2 v-1, u+v-1\right.\right.$ or $\left.\left.\frac{u+v-1}{2}\right\}\right)=(2 u-1)^{2}$

(since $\frac{1}{2}[d(u, T v)+d(v, T u)]=u+v-1$ or $\frac{u+v-1}{2}$, according to $v \neq T u$ or $v=T u$ respectively ) and

$\phi\left(m_{2}(u, v)\right)=\phi\left(\max \{(d(u, v), d(v, T v)\})=\phi(\max \{u+v, 2 v-1\})=\frac{1}{2}\right.$.

Then

$\psi(d(T u, T v)) \leq \psi\left(m_{1}(u, v)\right)-\phi\left(m_{2}(u, v)\right)$.

(ii) If $u \neq 0$ and $v>u$, then

$\psi(d(T u, T v))=\psi(d(u-1, v-1))=\psi(u+v-2)=(u+v-2)^{2}$,

$\psi\left(m_{1}(u, v)\right)=\psi\left(\max \left\{d(u, v), d(u, T u), d(v, T v), \frac{1}{2}[d(u, T v)+d(v, T u)]\right\}\right)$

$=\psi\left(\max \left\{u+v, 2 u-1,2 v-1, u+v-1\right.\right.$ or $\left.\left.\frac{u+v-1}{2}\right\}\right)=(2 v-1)^{2}$

(Since $\frac{1}{2}[d(u, T v)+d(v, T u)]=u+v-1$ or $\frac{u+v-1}{2}$, according to $u \neq T v$ or $u=T v$ respectively) and

$\phi\left(m_{2}(u, v)\right)=\phi\left(\max \{(d(u, v), d(v, T v)\})=\phi(\max \{u+v, 2 v-1\})=\frac{1}{2}\right.$.

Then

$\psi(d(T u, T v)) \leq \psi\left(m_{1}(u, v)\right)-\phi\left(m_{2}(u, v)\right)$.

(iii) If $v=0$, then

$\psi(d(T u, T v))=\psi(d(u-1,0))=\psi(u-1)=(u-1)^{2}$,

$\psi\left(m_{1}(u, v)\right)=\psi\left(\max \left\{d(u, v), d(u, T u), d(v, T v), \frac{1}{2}[d(u, T v)+d(v, T u)]\right\}\right)$

$=\psi\left(\max \left\{d(u, 0), d(u, T u), d(0,0), \frac{1}{2}[d(u, 0)+d(0, T u)]\right\}\right)$

$=\psi\left(\max \left\{u, 2 u-1,0, u-\frac{1}{2}\right\}\right)=(2 u-1)^{2}$

and

$\phi\left(m_{2}(u, v)\right)=\phi\left(\max \{(d(u, v), d(v, T v)\})=\phi\left(\max \{(d(u, 0), d(0,0)\})=\phi(u)=\frac{1}{2}\right.\right.$.

Then, $\psi(d(T u, T v)) \leq \psi\left(m_{1}(u, v)\right)-\phi\left(m_{2}(u, v)\right)$.

(iv) If $u=0$, then

$\psi(d(T u, T v))=\psi(d(0, v-1))=\psi(v-1)=(v-1)^{2}$,

$\psi\left(m_{1}(u, v)\right)=\psi\left(\max \left\{d(u, v), d(u, T u), d(v, T v), \frac{1}{2}[d(u, T v)+d(v, T u)]\right\}\right)$

$=\psi\left(\max \left\{d(0, v), d(0,0), d(v, T v), \frac{1}{2}[d(0, T v)+d(v, 0)]\right\}\right)$

$=\psi\left(\max \left\{v, 0,2 v-1, v-\frac{1}{2}\right\}\right)=(2 v-1)^{2}$ 


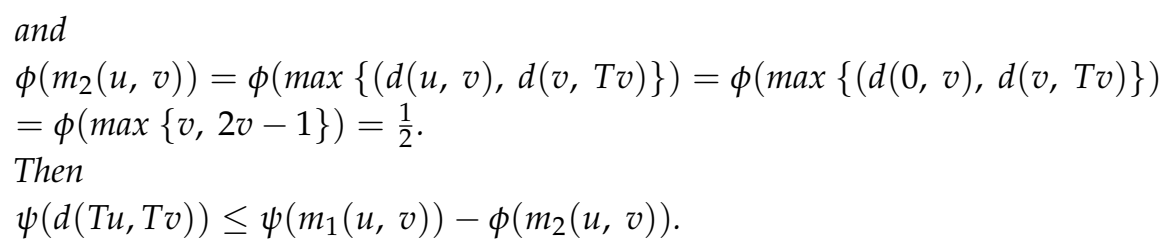

Case-II: $u=v$.

Then condition(vi) of Theorem 1 is trivially satisfied.

Therefore, from the above two cases, we have

$\psi(d(T u, T v)) \leq \psi\left(m_{1}(u, v)\right)-\phi\left(m_{2}(u, v)\right)$ for all $u, v \in U$, where $(u, v) \in \mathcal{R}$, $m_{1}(u, v)=\max \left\{d(u, v), d(u, T u), d(v, T v), \frac{1}{2}[d(u, T v)+d(v, T u)]\right\}$

and $m_{2}(u, v)=\max \{d(u, v), d(v, T v)\}$

Then the conditions of Theorem 1 hold in this case.

Here we see that 0 is a fixed point of $T$.

Further we see that $T(X)$ is $\mathcal{R}$-connected and $\mathcal{R}$ is $T$-transitive .

Hence the condition (vii.b) of Theorem 2 holds in this case.

Here it is seen that 0 is the unique fixed point for $T$.

Remark 4. (i) In the above example the relation $\mathcal{R}$ has reflexive and transitive properties as well which are not requirements of the theorem. However a weaker criterion of local transitivity is used here to produce the uniqueness.

(ii) In the above example if we put $u=-2$ and $v=-1$, then

$$
\begin{aligned}
\psi(d(T u, T v)) & =\psi(d(T(-2), T(-1))) \\
& =\psi(d(-2-1,-1-1))=\psi(d(-3,-2))=\psi(|-3|+|-2|)=\psi(5)=25, \\
m_{1}(u, v) & =m_{1}(-2,-1) \\
& =\max \left\{d(-2,-1), d(-3,-2), d(-1,-2), \frac{1}{2}[d(-2,-2)+d(-1,-3)]\right\} \\
& =\max \{3,5,3,2\} \\
& =5, \\
m_{2}(u, v) & =m_{2}(-2,-1), \\
& =\max \{d(-2,-1), d(-1,-2)\}, \\
& =\max \{3,3\} \\
& =3 .
\end{aligned}
$$

Thus we obtain, $\psi\left(m_{1}(u, v)\right)=5^{2}=25$ and $\phi\left(m_{2}(u, v)\right)=\phi(3)=\frac{1}{2}$.

Therefore in this case, the inequality of Equation (1) is not satisfied.

This shows that the inequality in Equation (1) does not hold for an arbitrary pair of points. Thus the result of [4] is not applicable to the present example.

\section{Conclusions}

The present work is in line with research exploring fixed point properties of various types of contractions under restrictions imposed by relations introduced in the original space on which the function is defined. We consider a type of weakly contractive mapping for our purpose which belongs to a class of mappings meant for weakening the corresponding contraction condition. Due to their less restrictive nature, the fixed point theorems obtained in metric space using relations have more scopes of applications. More precisely, they fit better for modeling the practical real world situations 
by the less requirements they have in their statements. Some applications are in works like [32,34]. Applications and real-world illustrations on similar lines can be explored for our present result and also for similar other results in future works. Applications may be attempted in areas of differential equations, integral equations, global optimizations, equilibrium problems, etc.. Higher dimensional illustrations of the present theorem can also be attempted.

We have already noted that the main result is an actual generalization of a previous result in [4]. The methodology here is comparable with several other similar results dealing with contractive inequalities noted in [3-10]. There are possibilities of applying relation-theoretic methods to other weakly contractive mappings. These problems can be taken up for future research.

Author Contributions: Conceptualization, P.C. and B.S.C.; methodology, B.S.C.; validation, B.S.C. and M.D.1.S.; formal analysis, P.C. and B.S.C.; investigation, P.C.; resources, P.C. and B.S.C.; writing-original draft preparation, P.C.; writing-review and editing, P.C., B.S.C. and M.D.1.S.; visualization, M.D.1.S.; supervision, M.D.1.S.; project administration, M.D.1.S.; funding acquisition, M.D.1.S. All authors have read and agreed to the published version of the manuscript.

Funding: This research received funding from the Basque Government through project IT1207-19.

Acknowledgments: The first author acknowledges the Indian Institute of Engineering Science of Technology, Shibpur, for supporting him as JRF. This work was also supported in part by the Basque Government through project IT1207-19.

Conflicts of Interest: The authors declare no conflicts of interest.

\section{References}

1. Alber, Y.I.; Guerre-Delabriere, S. Principles of weakly contractive maps in Hilbert spaces. In New Results in Operator Theory and Its Applications; Goldberg, I., Lyubich, Y., Eds.; Birkhäuser Verlag: Basel, Switzerland, 1997; Volume 98, pp. 7-22.

2. Rhoades, B.E. Some theorems on weakly contractive maps. Nonlinear Anal. Theory Methods Appl. 2001, 47, 2683-2693. [CrossRef]

3. Cho, S. Fixed point theorems for generalized weakly contractive mappings in metric spaces with applications. Fixed Point Theory Appl. 2018, 2018. [CrossRef]

4. Choudhury, B.S.; Konar, P.; Rhoades, B.E.; Metiya, N. Fixed point theorems for generalized weakly contractive mappings. Nonlinear Analysis. Theory Methods Appl. 2011, 74, 2116-2126. [CrossRef]

5. Choudhury, B.S.; Metiya, N.; Maniu, G.; Konar, P. Best proximity results: Optimization by approximate solutions. Fixed Point Theory Appl. 2016, 2016, 79. [CrossRef]

6. Choudhury, B.S.; Metiya, N.; Postolache, M. A generalized weak contraction principle with applications to coupled coincidence point problems. Fixed Point Theory Appl. 2013, 2013, 152. [CrossRef]

7. Choudhury, B.S.; Metiya, N.; Postolache, M.; Konar, P. A discussion on best proximity point and coupled best proximity point in partially ordered metric spaces. Fixed Point Theory Appl. 2015, 2015, 170. [CrossRef]

8. Dutta, P.N.; Choudhury, B.S. A generalization of contraction principle in metric spaces. Fixed Point Theory Appl. 2008. [CrossRef]

9. Jeong, G.S.; Rhoades, B.E. More maps for which $F(T),=F\left(T^{n}\right)$. Demonstratio Math. 2007, 40, 671-680. [CrossRef]

10. Kannan, R. Some results on fixed points-II. Amer. Math. Mon. 1969, 76, 405-408.

11. Sridarata, P.; Suantai, S. Common Fixed Point Theorems for Multi-Valued Weak Contractive Mappings in Metric Spaces with Graphs. Filomat 2018, 32, 671-680. [CrossRef]

12. Reich, S. Some remarks concerning contraction mappings. Can. Math. Bull. 1971, 14, 121-124. [CrossRef]

13. Salazar, L.A.; Reich, S. A remark on weakly contractive mappings. J. Nonlinear Convex Anal. 2015, 16, 767-773.

14. Harjani, J.; Sadarangani, K. Fixed point theorems for weakly contractive mappings in partially ordered sets. Nonlinear Analy. Theory Methods Appl. 2009, 71, 3403-3410. [CrossRef]

15. Nieto, J.J.; Lopez, R.R. Contractive mapping theorems in partially ordered sets and applications to ordinary differential equations. Order 2005, 22, 223-239. [CrossRef]

16. Radenovic, S.; Kadelburg, Z. Generalized weak contractions in partially ordered metric spaces. Comput. Math. Appl. 2010, 60, 1776-1783. [CrossRef] 
17. Ran, A.C.M.; Reurings, M.C.B. A fixed point theorem in partially ordered sets and some application to matrix equations. Proc. Am. Math. Soc. 2004, 132, 1435-1443. [CrossRef]

18. Turinici, M. Fixed points for monotone iteratively local contractions. Demonstratio Math. 1986, 19, 171-180.

19. Turinici, M. Abstract comparison principles and multivariable Gronwall-Bellman inequalities. J. Math. Anal. Appl. 1986, 117, 100-127. [CrossRef]

20. Choudhury, B.S.; Metiya, N.; Debnath, P. End Point Results in Metric Spaces Endowed with a Graph. J. Math. 2016 2016, 1-7. [CrossRef]

21. Jachymski, J. The contraction principle for mappings on a metric space with a graph. Proc. Am. Math. Soc. 2008, 136, 1359-1373. [CrossRef]

22. Beg, I.; Butt, A.R.; Radojevic, S. The contraction principle for set valued mappings on a metric space with a graph. Comput. Math. Appl. 2010, 60, 1214-1219. [CrossRef]

23. Ahmadullah, M.; Imdad, M.; Gubran, R. Relation-theoretic metrical fixed point theorems under nonlinear contractions. Fixed Point Theory 2019, 20, 3-18. [CrossRef]

24. Alama, A.; Imdad, M. Relation-Theoretic Metrical Coincidence Theorems. Filomat 2017, 31, 4421-4439. [CrossRef]

25. Alam, A.; Imdad, M. Relation-theoretic contraction principle. J. Fixed Point Theory Appl. 2015, 17, 693-702. [CrossRef]

26. Alam, A.; Imdad, M. Nonlinear contractions in metric spaces under locally T-transitive binary relations. Fixed Point Theory 2018, 19, 13-24. [CrossRef]

27. Reich, S.; Zaslavski, A.J. Generic well-posedness of the fixed point problem for monotone nonexpansive mappings. In Mathematics Almost Everywhere; World Scientific: Hackensack, NJ, USA, 2018; pp. 169-179.

28. Rhoades, B.E. A comparison of various definitions of contractive mappings. Trans. Am. Math. Soc. 1977, 226, 257-290. [CrossRef]

29. Lipschutz, S. Schaum's Outlines of Theory and Problems of Set Theory and Related Topics; McGraw-Hill: New York, NY, USA, 1964.

30. Maddux, R.D. Relation Algebras. In Studies in Logic and the Foundations of Mathematics; Elsevier B. V.: Amsterdam, The Netherlands, 2006; p. 150.

31. Turinici, M. Product fixed points in ordered metric spaces. arXiv 2011, arXiv:1110.3079v1.

32. Samet, B.; Turinici, M. Fixed point theorems on a metric space endowed with an arbitrary binary relation and applications. Commun. Math. Anal. 2012, 13, 82-97.

33. Kolman, B.; Busby, R.C.; Ross, S. Discrete Mathematical Structures, 3rd ed.; PHI Pvt. Ltd.: New Delhi, India, 2000.

34. Perveen, A.; Khan, I.A.; Imdad, M. Relation Theoretic Common Fixed Point Results for Generalized Weak Nonlinear Contractions with an Application. Axioms 2019, 8, 49. [CrossRef]

(C) 2019 by the authors. Licensee MDPI, Basel, Switzerland. This article is an open access article distributed under the terms and conditions of the Creative Commons Attribution (CC BY) license (http://creativecommons.org/licenses/by/4.0/). 